\title{
Actividad física en chicas adolescentes con riesgo de desarrollar un trastorno alimentario
}

\section{Physical activity in adolescent girls at risk of developing an eating disorder}

Fecha de recepción: 17-06-2019

Fecha de aceptación: 15-11-2019
Lidia Pamies-Aubalat

Universidad Católica de Murcia

Yolanda Quiles Marcos

Universidad Miguel Hernández de Elche

\section{resumen/abstract:}

La literatura ha manifestado que la actividad física puede actuar como un factor de riesgo y/o un factor mantenedor en los trastornos alimentario (TA). El objetivo de este trabajo fue analizar la relación entre la práctica de actividad física con el riesgo de desarrollar un TA, analizando la frecuencia, intensidad y duración, el tipo de actividad y los motivos de realización de esa actividad. Método: la muestra estuvo compuesta por 1130 chicas adolescentes entre 11 y 19 años ( $M$ = 13.94; $D T=1.35)$; cumplimentaron el Eating Attitude Test (EAT-40), la subescala de insatisfacción corporal del Eating Disorder Inventory (EDI-2) y el Inventario de Conductas de Salud en Escolares. Resultados: Las adolescentes con menor riesgo de desarrollar un TA presentaron puntuaciones medias superiores en la realización de actividad con el motivo de divertirse, estar en buena forma y ver amigos que las adolescentes con riesgo de desarrollar un TA, que presentaron puntuaciones superiores en la práctica de actividad física para perder peso y quemar calorías. Conclusiones: conocer las características de la práctica de actividad física relacionada con los TA, permite generar propuestas preventivas eficaces, impedir que estos trastornos se mantengan e incluso se cronifiquen.

The literature has shown that physical activity can be a risk factor and / or a maintenance factor in eating disorders (ED). The objective of this research was to analyze the relationship between the practice of physical activity and the risk of developing an ED; Analyzing the frequency, intensity and duration, the type of activity and the reasons for carrying out that activity. Method: the sample consisted of 1130 adolescent girls between 11 and 19 years old $M=13.94, S D$ $=1.35)$; They completed the Eating Attitude Test (EAT-40), the body dissatisfaction subscale of the Eating Disorder Inventory (EDI-2) and the School Health Behavior Inventory. Results: The adolescents with lower risk of developing an ED had higher average scores in the performance of activity with the motive of having fun, being in good shape and seeing friends that the adolescents with risk of developing an ED, who had higher scores in practice of physical activity to lose weight and burn calories. Conclusions: knowing the characteristics of the practice of physical activity related to ED, allows to generate effective preventive proposals, prevent these disorders from being maintained and even become chronic.

\section{palabras clave/keywords:}

Actividad física, deporte, ejercicio físico, trastorno alimentario, adolescentes

Physical activity, sport, exercise, eating disorder, adolescents 


\section{Introducción}

Los Trastornos Alimentarios (TA) más prevalentes en población adolescente y joven son: la anorexia nerviosa, la bulimia nerviosa, el trastorno por atracón y el trastorno alimentario no especificado. Según el DSM-5 (APA, 2013), estos trastornos se caracterizan por presentar alteraciones en la alimentación, una distorsión de la imagen corporal y por causar graves consecuencias sobre la salud y la vida de las personas que la padecen. Respecto a su prevalencia, uno de los últimos estudios realizados en población española, pone de manifiesto que, la prevalencia global de los TA se sitúa en el $4.11 \%$ (5.46\% mujeres y $2.55 \%$ hombres). Si se atiende al tipo de diagnóstico, señalan un $0.19 \%$ para la anorexia, un $0.57 \%$ para la bulimia y un 3.34\% para el TA no especificado (Álvarez-Malé, Bautista y Serra, 2015).

La etiopatogenia de los TA es multicausal, hay consenso en afirmar que en el desarrollo de estos trastornos existen diferentes factores de riesgo: biológicos, psicológicos, sociales y familiares (Benavente, Rodríguez, Martin e Hiruela, 2003; Alfoukha, Hamdan y Banihani, 2019). Entre estos se ha destacado que la actividad física puede tener un papel relevante en el desarrollo de estos trastornos (Allain y Lentillan, 2016; Thome y Espelage, 2004).

La realización de actividad física se considera una conducta saludable si se realiza de una manera regular y moderada (Castillo, Balaguer y García-Merita, 2007). Sin embargo, desde aproximadamente la década de los 70 , la realización de actividad física de una forma abusiva y practicada con gran intensidad ha sido descrita como un factor relevante en la etiología, desarrollo y mantenimiento de los TA (Alonso, 2006; Durán, Jiménez, Ruiz, Jiménez y Camacho, 2006). Es así que la actividad física, ha sido considerada, no solo un síntoma característico de los TA (excesiva actividad con la finalidad de perder peso y modificar su cuerpo), sino también como un factor importante en el desarrollo de estos trastornos. Así, por ejemplo, en un estudio llevado a cabo recientemente se analizó la relación entre la práctica de actividad física y actitudes alimentarias anómalas en adolescentes de ambos géneros. Los resultados pusieron de manifiesto que los jóvenes que realizaban ejercicios de tonificación y fuerza 7 días a la semana informaban de más vómitos, uso de laxantes y pastillas para adelgazar que los adolescentes que realizaban este ejercicio de 0 a 3 días por semana (Wang et al., 2013).

Por otro lado, la investigación de Peñas-Lledó, Sancho y Waller (2002) tuvo como objetivo conocer la relación entre el riesgo de TA y la frecuencia con la que se realizaba la actividad en jóvenes pre-universitarios. Los resultados mostraron diferencias significativas en función del género. Así, las chicas que realizaban actividad física de forma regular presentaban mayores puntuaciones en el EDI (Eating Disoder Inventory), en comparación con las chicas que realizaban actividad física de forma esporádica, por lo que mostraban un mayor riesgo de desarrollar un TA.

En relación a los tipos de práctica de actividad física, por un lado, podemos clasificar su práctica entre la realización de ejercicio físico y deporte, entendiendo como ejercicio físico a la actividad física ordenada, planificada y repetitiva dirigida a la mejora de la condición física. En cambio, el deporte incluye la competición, el sometimiento de reglas establecidas y la existencia de organizaciones estructuradas que regulan su práctica. Esta diferenciación 
permite establecer relaciones con la calidad de vida y salud de las personas (Cantó, 2001). En esta línea, Dosil (2003) realizó una clasificación de aquellos deportes que deben tener una mayor vigilancia, por relacionarse con el peso y caracterizarse por el hecho de estar delgados y, por tanto, relacionarse con el riesgo de TA. Diferenció entre deportes de estética, en los que la estética corporal exige delgadez (patinaje, danza, gimnasia rítmica y artística, etc.); deportes de categorías, donde el peso es determinante para estar en una u otra categoría (judo, boxeo, lucha, etc.); deportes de gimnasio, donde se persigue aumentar musculatura y perder grasa corporal (culturismo, aerobic, fitness, etc.); y deportes de resistencia y requieren un cuerpo delgado para rendir más (atletismo, natación, ciclismo, etc.).

Atendiendo a esta clasificación, French, Perry, León y Fulkerson (1994) analizaron la relación entre los diferentes tipos de actividad física practicada y el riesgo de desarrollar un TA. En este trabajo participaron 1494 adolescentes de ambos géneros y se agrupó la actividad física en diferentes tipos: en condicionales (correr, levantamiento de pesas, etc.), deportes atípicos (aerobic, gimnasia, bailar) y deportes de ocio y tiempo libre (nadar, patinar, navegar). En las chicas, los deportes condicionales y los deportes atípicos explicaron en positivo el riesgo de desarrollar un TA; y los deportes de ocio y tiempo lo explicaron en sentido negativo. En esta línea, la investigación desarrollada por Kjelsas y Augestad (2004) puso de manifiesto que en las chicas, el riesgo de desarrollar un TA se relacionaba de forma negativa con practicar deportes de pelota.

Cuando se tiene en cuenta los motivos de práctica de actividad física, la literatura indica que el riesgo de desarrollar un TA se relaciona positivamente con realizar actividad física para sentirse atractivo, para mejorar la salud y estar en forma. Mientras se relaciona de forma negativa con el objetivo de mantener el peso (Furnham, Badmin y Sneade, 2002; Adkins y Keel, 2005). Similares resultados obtuvieron Allain y Lentillan (2016) en su investigación con 1270 mujeres, en los que los motivos de perder peso y mejorar su apariencia física se relacionaron con un mayor riesgo de desarrollar el TA y una mayor insatisfacción corporal.

Una vez expuesta la relación entre el riesgo de desarrollar un TA y la práctica de actividad física, el objetivo de este trabajo fue analizar dicha relación en mujeres adolescentes, considerando la frecuencia e intensidad, tipo y motivos de realización de actividad física.

\section{Material y métodos \\ Participantes}

La población de interés estuvo formada por todos los centros educativos de secundaria (privados, concertados y públicos) de la provincia de Alicante (España). Se utilizó un muestreo por conglomerados monoetápico. Para obtener una muestra de estudiantes de secundaria representativa de la población, se requería un tamaño muestral de 2142 estudiantes, la cual se obtuvo con el total de alumnos matriculados en 9 de los centros extraídos aleatoriamente del total, con un nivel de confianza del $95 \%$ y un error de estimación de \pm 2 .

Finalmente, en el estudio participaron 9 centros educativos de la provincia de Alicante (5 públicos, 1 privado y 3 concertados). En este estudio participaron finalmente 1130 chicas, de las cuales el $77.88 \%(\mathrm{n}=880)$ iban a un colegio público, el $15.66 \%(\mathrm{n}=177)$ a un cole- 
gio concertado y el $5.75 \%(n=65)$ a un colegio privado. Estas chicas tenían una edad media de 13.94 años $(D T=1.35)$. Cursaban $1^{\circ}$ ESO el $27.34 \%(n=309), 2^{\circ}$ Eso el $27.70 \%(n=$ $313), 3^{\circ}$ ESO el $29.72 \%(n=263)$ y $4^{\circ}$ ESO el $21.68 \%(n=245)$.

Además, se obtuvo el Índice de Masa Corporal (IMC) en las chicas que participaron en el estudio. Este IMC fue clasificado siguiendo las indicaciones de la Organización Mundial de la Salud (OMS). Los porcentajes de adolescentes en cada una de las categorías establecidas por la OMS, pusieron de manifiesto que la mayoría de las participantes en el estudio se encontraban en normopeso (61.9\%), así como, el $22.6 \%$ estaba en bajo peso e infrapeso y el $11 \%$ con sobrepeso y obesidad.

\section{Instrumentos}

Cuestionario de variables sociodemográficas (ad hoc); mediante el que se obtuvo información sobre edad, género y curso escolar.

Variables antropométricas; se calculó el IMC de las adolescentes obtenido a partir de la fórmula: (peso en $\mathrm{kg} /$ talla en $\mathrm{m}^{2}$ ) . Para lo cual, se procedió a pesar y medir en el momento de la recogida de datos a las participantes.

Eating Attitude Test (EAT-40) (Garner y Garfinkel, 1979), adaptado a la población española por Castro et al. (1991) y posteriormente validado como prueba de cribado de los TA en población general por Irala et al. (2008). Se trata de un cuestionario autoadministrado diseñado para detectar la presencia de actitudes alimentarias anómalas, especialmente las relacionadas con el miedo a ganar peso, el impulso de adelgazar y la presencia de patrones alimentarios restrictivos. Consta de 40 ítems que se valoran en una escala de frecuencia tipo Likert de 6 puntos, desde nunca a siempre, y se puntúa entre 0 y 3. La adaptación española del cuestionario establece tres factores: restricción alimentaria y preocupación por los alimentos, presión social percibida y malestar ante la ingesta y trastornos psicobiológicos. La fiabilidad de la escala en el trabajo original de la adaptación española fue igual a .93 y, en este trabajo, de .83 .

Subescala de Insatisfacción corporal, del Eating Disorder Investory 2 (EDI-2) (Garner, 1998). Este instrumento consta de 11 subescalas, entre ellas la de insatisfacción corporal, que pueden utilizarse separadamente con buenos índices de fiabilidad. Consta de 9 ítems con una escala de respuesta de seis puntos desde "nunca" a "siempre", obteniendo puntuaciones que oscilan entre 0 y 27. En este estudio, presentó una consistencia interna $\alpha=.87$.

Inventario de Conductas de Salud en Escolares (The Health Behavior in Schoolchildren 1985/86: A WHO crossnational survey, Wold, 1995) adaptado a población española (Balaguer, 1999; Balaguer, 2002). Este instrumento fue diseñado por la oficina regional europea de la OMS para el desarrollo de un programa de investigación internacional sobre los estilos de vida de los adolescentes. Los ítems de este cuestionario que se han utilizado en este estudio son aquellos que hacen referencia a la evaluación de la actividad física. Concretamente, se preguntaba sobre el tipo de realización de actividad física fuera del horario extraescolar, frecuencia con la que se practicaba, utilizando un ítem con una escala Likert de 4 puntos, entre "Todos los días de la semana" y "nunca"; también, se preguntó por la duración e 
intensidad de la realización de actividad física en una escala Likert de 3 puntos. Además, este cuestionario permite saber si la actividad física que realizan se trata de ejercicio físico o bien de deporte. También, se evaluó si se realizaba actividad física de forma individual y grupal; así como, cuáles eran los motivos que los llevaban a practicar la actividad física, por ejemplo, "para divertirme", "para ver a mis amigos" o "para quemar calorías". Evaluando seis motivos de práctica de actividad física en una escala Likert de 4 puntos, que iba desde entre "nada" a "siempre", oscilando la puntuación en cada una de ellas entre 0 y 4.

\section{Procedimiento}

En primer lugar, se llevó a cabo una entrevista con los directores y orientadores de los centros participantes para exponer los objetivos de la investigación, solicitar permiso y promover su colaboración. Tras la aceptación por parte de los centros educativos para participar en la investigación, se envió una circular a los padres de los estudiantes en la que se les informaba de la investigación que se iba a realizar, y se solicitaba el consentimiento informado por escrito autorizando a sus hijas a participar en el estudio. Los cuestionarios fueron administrados de forma colectiva, anónima y voluntaria en horario de clase. Los investigadores estuvieron presentes durante la administración de los cuestionarios para proporcionar ayuda si era necesaria, para verificar la cumplimentación correcta e independiente por parte de los sujetos.

\section{Análisis de datos}

Para realizar los análisis estadísticos se ha utilizado el programa SPSS V.24.0 (2016). Se han realizado los análisis descriptivos de las variables de estudio. Para estudiar la relación entre la práctica de actividad física con el riesgo de desarrollar un TA se utilizó el análisis de correlación de Pearson. Por otro lado, las diferencias entre medias se utilizó la t-Student como prueba paramétrica para dos muestras independientes. Siguiendo las recomendaciones de Cohen (1998), hemos considerado el tamaño del efecto, para evitar el sesgo de los tamaños muestrales. Así hemos utilizado el índice $d$, que nos indica hasta qué punto una significación estadística es relevante eliminando el efecto del tamaño muestral. Cohen clasificó el tamaño del efecto como pequeño $(\mathrm{d} \approx 0.20)$, medio $(\mathrm{d} \approx 0.50)$ y grande $(\mathrm{d} \approx 0.80)$. Además, se ha realizado la ANOVA de un factor como prueba paramétrica de K muestras independientes y procedimiento Bonferroni para comparaciones múltiples. Así mismo, para las variables categóricas se han calculado las diferencias a partir de tablas de contingencia y el estadístico chi-cuadrado.

\section{Resultados}

En primer lugar, se realizaron análisis descriptivos para analizar las características de la muestra en las diferentes variables de estudio (ver tabla 1). En referencia al riesgo de desarrollo de un TA, evaluado a partir del instrumento EAT - 40, la puntuación media fue de $17.43(D T=12.24)$, atendiendo al punto de corte de 30 propuesto por los autores originales de la escala, en este estudio el $15 \%$ de las chicas presentó riesgo de desarrollar un TA. Así mismo, en referencia a la realización de actividad física, en la tabla 1 se describe la media obtenida en frecuencia, duración e intensidad de realización, así como, de los motivos por los que practican actividad física. 


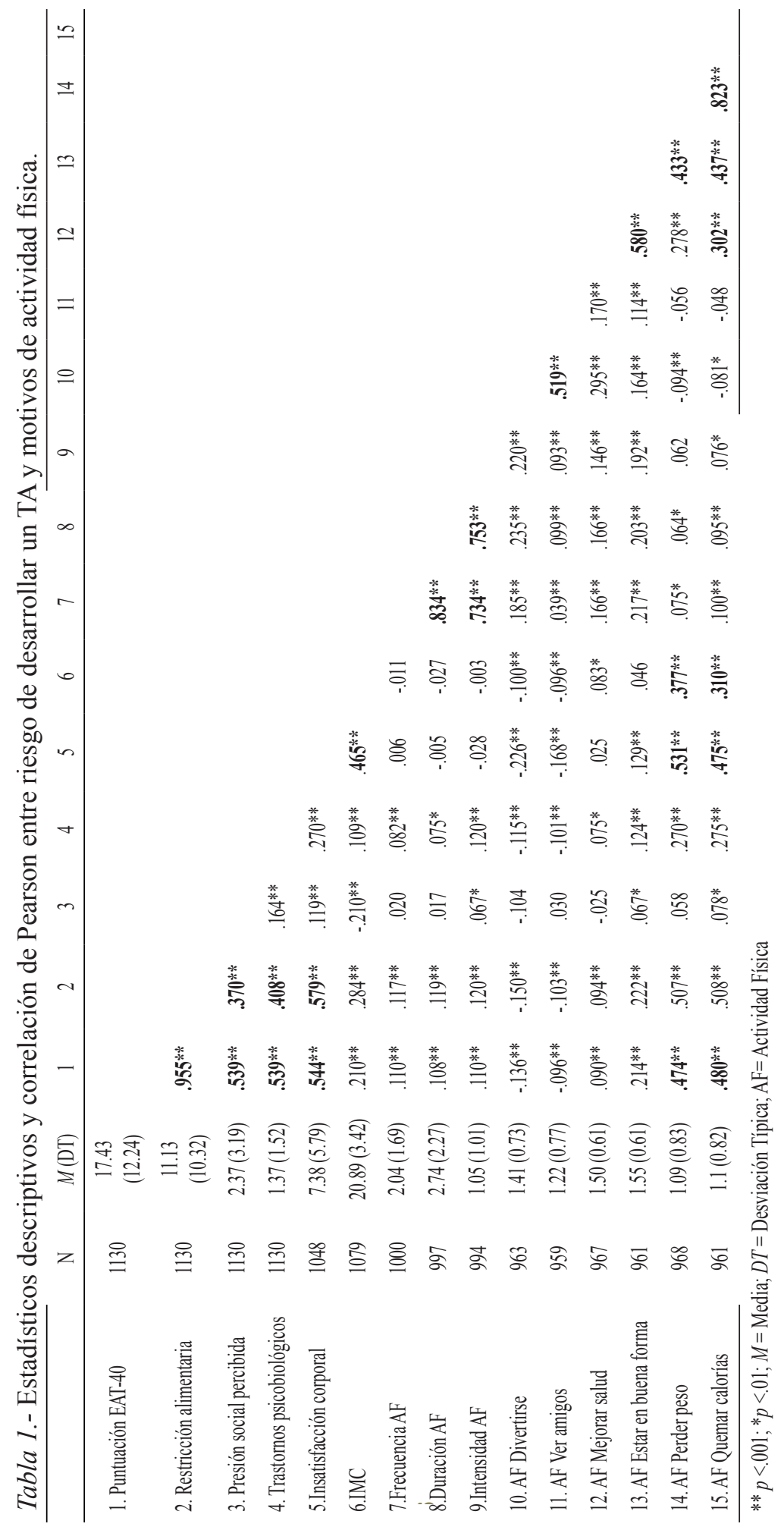


Así mismo, los análisis descriptivos de frecuencias de la realización de actividad física, ponen de manifiesto que el $35.1 \%(\mathrm{n}=553)$ de las chicas manifestó realizar algún tipo de actividad física. De los que manifestaron realizarla, el $11.8 \%$ lo realizaba sola, el $61.2 \%$ con otras personas y el $27 \%$ unas veces sola y otras veces en compañía. Además, el $81.8 \%$ realizaba sólo un tipo de actividad física y el $18.2 \%$ más de dos.

En segundo lugar, se realizaron los análisis de correlación entre las variables relacionadas con el riesgo de desarrollar un TA (puntuación total EAT-40, factores del EAT-40, insatisfacción corporal e IMC) y la práctica de actividad física (frecuencia, duración e intensidad y motivos) (ver tabla 1). Los análisis mostraron que la realización de actividad física con el motivo de perder peso, presentó relaciones positivas y significas con la puntuación total en el EAT-40 $(r=.474 ; p<.001)$, la insatisfacción corporal $(r=.531 ; p<.001)$ y el IMC $(r$ $=.377 ; p<.001)$. Resultados similares se obtuvieron con la realización de actividad física con el propósito de quemar calorías, que se relacionó positivamente con la puntuación total del EAT-40 $(r=.480 ; p<.001)$, la insatisfacción corporal $(r=.475 ; p<.001)$ y el IMC $(r=$ $.310 ; p<.001)$.

A continuación, se analizó si existían diferencias en el riesgo de desarrollar un TA entre las adolescentes que realizaban o no alguna actividad física, en función de la práctica de si se realiza o no algún tipo de práctica de actividad física (ver tabla 2). Los análisis mostraron que las chicas que no practicaban actividad física tenían puntuaciones medias más bajas en el EAT- $40(M=15.95 ; D T=10.92)$ que las chicas que sí practicaban $(M=18.24 ; D T=$ 13.05), siendo esta diferencia significativa, aunque el tamaño del efecto fue bajo $(t=-2.94$; $p<.001 ; d=.18)$.

Por otro lado, se analizó si existían las diferencias entre las chicas con riesgo o no riesgo de desarrollar un TA en función de si practicaban la actividad física sola o en compañía, para

Tabla 2.- Análisis de diferencias entre riesgo de TA y realización de actividad física

\begin{tabular}{|c|c|c|c|c|c|c|}
\hline & Actividad fisica & $N$ & $M$ & DT & $t$ & $d$ \\
\hline \multirow[t]{2}{*}{ Puntuación EAT-40 } & No & 353 & 15.95 & 10.92 & \multirow{2}{*}{$-2.94 * *$} & \multirow{2}{*}{.18} \\
\hline & Sí & 652 & 18.24 & 13.05 & & \\
\hline \multirow[t]{2}{*}{ Restricción alimentaria y preocupación } & No & 353 & 9.83 & 9.09 & \multirow{2}{*}{$-3.08 * *$} & \multirow{2}{*}{.19} \\
\hline & Sí & 652 & 11.83 & 11.09 & & \\
\hline \multirow[t]{2}{*}{ Restricción alimentaria y preocupación } & No & 353 & 2.25 & 3.26 & \multirow{2}{*}{$-0.64 n s$} & \\
\hline & Sí & 652 & 2.38 & 3.16 & & \\
\hline \multirow[t]{2}{*}{ Presión social percibida y malestar } & No & 353 & 1.23 & 1.46 & \multirow{2}{*}{$-2.35^{*}$} & \multirow{2}{*}{.15} \\
\hline & Sí & 652 & 1.46 & 1.56 & & \\
\hline \multirow[t]{2}{*}{ Insatisfacción corporal } & No & 350 & 7.53 & 5.66 & \multirow{2}{*}{$0.72 n s$} & \\
\hline & Sí & 648 & 7.25 & 5.89 & & \\
\hline \multirow[t]{2}{*}{ Índice Masa Corporal } & No & 341 & 21.06 & 3.19 & \multirow{2}{*}{$1.64 n s$} & \\
\hline & Sí & 637 & 20.71 & 3.37 & & \\
\hline
\end{tabular}

${ }^{* *} \mathrm{p}<.01 ;{ }^{*} \mathrm{p}<.05 ; M=$ Media; $D T=$ Desviación Típica; $t=$ estadístico t-studen; $d=$ Tamaño del efecto de Cohen 
ello, al tratarse de variables categóricas, se utilizaron tablas de contingencia (ver tabla 3). Los resultados que se presentan en la tabla 4 se muestran los porcentajes que resultaron ser estadísticamente significativos $(? 2=13.12(1) ; \mathrm{p}<.001 ; \mathrm{V}$ - Crammer $=.145 ; \mathrm{p}<.001)$.

En tercer lugar, se clasificó la actividad física que realizaban las chicas bien en "ejercicio físico" o "deporte", para posteriormente analizar si existían diferencias en el riesgo de de-

Tabla 3.- Análisis de contingencia entre realización de actividad física y riesgo TA

\begin{tabular}{llcc}
\hline \multirow{2}{*}{ Realización actividad física } & & \multicolumn{2}{c}{ Riesgo TA } \\
\hline Con otros & & No riesgo & Riesgo \\
& Frecuencia & 326 & 57 \\
En solitario & Porcentaje & $85.11 \%$ & $14.88 \%$ \\
& Frecuencia & 51 & 23 \\
Con otros y en solitario & Porcentaje & $68.92 \%$ & $31.08 \%$ \\
& Frecuencia & 146 & 23 \\
\hline
\end{tabular}

sarrollar un TA en función de si las chicas manifestaron realizar deporte o ejercicio físico. Estos análisis estadísticos no mostraron diferencias significativas $(t=-3.15 ; p=.75)$. Cuando se analizó si existían diferencias significativas en los motivos de práctica de actividad física en función de si las adolescentes realizaban ejercicio físico o deporte, los resultados mostraron diferencias. Las chicas que practicaban deporte lo hacían en mayor medida para ver a sus amigos $(t=5.00 ; p<.001 ; d=.37)$ y divertirse $(t=5.00 ; p<.001 ; d=.27)$, que las que realizaban ejercicio físico. En cambio, las que realizaban ejercicio lo hacían en mayor medida con el objetivo de perder peso $(t=5.00 ; p<.001 ; d=.24)$. También, en lo referente a duración de la actividad física, las chicas que practicaban deporte le dedicaban más tiempo $(M=4.60 ; D T=0.96)$ que las que realizaban ejercicio físico $(M=3.79 ; D T=1.48)$ de manera significativa $(t=8.09 ; p<.001 ; d=.67)$. Además, las chicas que practicaban deporte lo hacían de manera más intensa $(M=1.76 ; D T=0.80)$ que las que practicaban ejercicio físico $(M=1.43 ; D T=0.80)$, siendo esta diferencia significativa $(t=5.00 ; p<.001 ; d=.41)$.

Aquellas adolescentes que indicaron que realizaban algún tipo de actividad física, fueron agrupadas según la clasificación propuesta por Dosil (2003), cuyas frecuencias y porcentajes quedan representados en la tabla 5.

Se calculó si existían diferencias en el riesgo de desarrollar un TA en función del tipo de deporte practicado, y los resultados no mostraron diferencias significativas $(F=0.17 ; p=.95)$. No obstante, si se encontraron diferencias respecto a la insatisfacción corporal y al IMC. Respecto a la insatisfacción corporal, aquellas chicas que realizaban deportes de gimnasio $(M=0.89 ; D T=0.03)$, presentaban mayor insatisfacción corporal que las que practicaban 
Tabla 4.- Análisis de diferencias entre tipo de actividad física y motivos para practicarla

\begin{tabular}{|c|c|c|c|c|c|c|}
\hline & Actividad física & $\mathrm{N}$ & Media & DT & $\mathrm{T}$ & $d$ \\
\hline \multirow{2}{*}{ AF Divertirse } & Deporte & 354 & 1.63 & 0.57 & \multirow{2}{*}{$4.59 * * *$} & \multirow{2}{*}{.37} \\
\hline & Ejercicio & 246 & 1.39 & 0.75 & & \\
\hline \multirow{2}{*}{ AF Ver amigos } & Deporte & 349 & 1.34 & 0.72 & \multirow{2}{*}{$3.19^{* *}$} & \multirow{2}{*}{.26} \\
\hline & Ejercicio & 245 & 1.15 & 0.77 & & \\
\hline \multirow{2}{*}{ AF Mejorar salud } & Deporte & 353 & 1.58 & 0.53 & \multirow{2}{*}{-0.08} & \multirow{2}{*}{-} \\
\hline & Ejercicio & 247 & 1.58 & 0.57 & & \\
\hline \multirow{2}{*}{ AF Estar en buena forma } & Deporte & 352 & 1.61 & 0.55 & \multirow{2}{*}{-1.57} & \multirow{2}{*}{-} \\
\hline & Ejercicio & 248 & 1.67 & 0.50 & & \\
\hline \multirow{2}{*}{ AF Perder peso } & Deporte & 351 & 1.03 & 0.84 & \multirow{2}{*}{$-2.86^{* *}$} & \multirow{2}{*}{.24} \\
\hline & Ejercicio & 250 & 1.23 & 0.80 & & \\
\hline \multirow{2}{*}{ AF Quemar calorías } & Deporte & 351 & 1.08 & 0.82 & \multirow{2}{*}{$-2.12^{*}$} & \multirow{2}{*}{.17} \\
\hline & Ejercicio & 246 & 1.22 & 0.77 & & \\
\hline
\end{tabular}

${ }^{* * *} p<.001 ;{ }^{* *} p<.01 ; * p<.05 ; M=$ Media; $D T=$ Desviación Típica; $t=$ estadístico t-studen; $d=$ Tamaño del efecto de Cohen; AF= Actividad Física

deportes de estética, como gimnasia rítmica y artística, patinaje, danza, etc. $(M=1.42 ; D T$ $=1)$, siendo esta diferencia significativa $(F=2.46 ; p<.05)$. En referencia al IMC, las chicas que practicaban un deporte de estética tenían un menor IMC $(M=19.69 ; D T=2.89)$, que las chicas que realizaban deportes por categorías $(M=22.46 ; D T=3.42)$, siendo significativa esta diferencia $(F=3.69 ; p<.01)$. Además, también se analizaron las diferencias en los motivos de practicar actividad física en función del tipo de deporte (ver tabla 5).

Para finalizar, se clasificó a las adolescentes en función del punto de corte del EAT-40, en chicas con riesgo y no riesgo de desarrollar un TA, con el fin de analizar las diferencias entre estos dos grupos en la práctica de actividad física (ver tabla 6).

Tabla 5.- Análisis de diferencias en los motivos de practica de AF y tipo de deporte

\begin{tabular}{|c|c|c|c|c|c|c|c|c|c|c|c|c|c|}
\hline & \multirow[b]{2}{*}{$\mathrm{N}(\%)$} & \multicolumn{2}{|c|}{ AF divertirse } & \multicolumn{2}{|c|}{$\begin{array}{c}\text { AF mejorar } \\
\text { salud }\end{array}$} & \multicolumn{2}{|c|}{$\begin{array}{l}\text { AF estar buena } \\
\text { forma }\end{array}$} & \multicolumn{2}{|c|}{$\begin{array}{l}\text { AF perder } \\
\text { peso }\end{array}$} & \multicolumn{2}{|c|}{$\begin{array}{l}\text { AF Quemar } \\
\text { calorías }\end{array}$} & \multicolumn{2}{|c|}{ AF ver amigos } \\
\hline & & $M$ & $D T$ & $M$ & $D T$ & $M$ & $D T$ & $M$ & $D T$ & $M$ & $D T$ & $M$ & $D T$ \\
\hline 1.Estética & $78(12.9 \%)$ & 1.58 & 0.63 & 1.56 & 0.55 & 1.64 & 0.60 & 1.01 & 0.86 & 1.05 & 0.83 & 1.16 & 0.73 \\
\hline 2.Categorías & $21(3.6 \%)$ & 1.71 & 0.56 & 1.81 & 0.40 & 1.81 & 0.40 & 1.10 & 0.83 & 1.10 & 0.83 & 1.43 & 0.60 \\
\hline 3.Gimnasio & $96(16.2 \%)$ & 1.26 & 0.76 & 1.59 & 0.59 & 1.68 & 0.47 & 1.38 & 0.71 & 1.34 & 0.69 & 1.11 & 0.84 \\
\hline 4.Resistencia & $115(19.4 \%)$ & 1.52 & 0.69 & 1.58 & 0.55 & 1.67 & 0.51 & 1.17 & 0.81 & 1.16 & 0.83 & 1.18 & 0.77 \\
\hline 5. Sin riesgo & $226(37.4 \%)$ & 1.69 & 0.51 & 1.56 & 0.53 & 1.59 & 0.55 & 1.03 & 0.85 & 1.08 & 0.82 & 1.40 & 0.70 \\
\hline \multicolumn{2}{|c|}{ Comparación grupos } & \multicolumn{4}{|c|}{$3<1,2,4$ y 5} & & & \multicolumn{2}{|c|}{$3>1$ y 5} & & & \multicolumn{2}{|c|}{$3<5$} \\
\hline \multicolumn{2}{|l|}{$\mathrm{F}$} & \multicolumn{2}{|c|}{$\mathrm{F}=8.52 * * *$} & \multicolumn{2}{|c|}{ n.s. } & \multicolumn{2}{|c|}{ n.s. } & \multicolumn{2}{|c|}{$\mathrm{F}=3.49^{* *}$} & \multicolumn{2}{|c|}{ n.s } & \multicolumn{2}{|c|}{$\mathrm{F}=4.07 * *$} \\
\hline
\end{tabular}

*** $p<.001 ; * * p<.01 M=$ Media DT= Desviación Típica $n . s .=$ no significativa 
Los resultados mostraron que no existían diferencias entre ambos grupos en la frecuencia, duración e intensidad con la que practicaban la actividad física. Respecto a los motivos, las chicas con riesgo consideraban más importante realizar actividad física para perder peso

Tabla 6. - Diferencias en la realización de actividad física en función del riesgo de desarrollar un TA

\begin{tabular}{|c|c|c|c|c|c|c|}
\hline & Riesgo TA & $\mathrm{N}$ & Media & DT & $\mathrm{T}$ & $d$ \\
\hline \multirow{2}{*}{ Frecuencia actividad física } & Riesgo & 151 & 2.28 & 1.77 & \multirow{2}{*}{1.90} & \multirow{2}{*}{-} \\
\hline & No riesgo & 849 & 1.99 & 1.67 & & \\
\hline \multirow{2}{*}{ Duración actividad física } & Riesgo & 150 & 3.05 & 2.23 & \multirow{2}{*}{1.83} & \\
\hline & No riesgo & 847 & 2.68 & 2.28 & & - \\
\hline \multirow{2}{*}{ Intensidad actividad física } & Riesgo & 150 & 1.19 & 1.06 & \multirow{2}{*}{1.89} & \multirow{2}{*}{-} \\
\hline & No riesgo & 844 & 1.02 & 1.00 & & \\
\hline \multicolumn{7}{|l|}{ Motivos actividad física } \\
\hline \multirow{2}{*}{ Divertirse } & Riesgo & 143 & 1.23 & 0.81 & \multirow{2}{*}{$-3.15^{*}$} & \multirow{2}{*}{0.2} \\
\hline & No riesgo & 820 & 1.44 & 0.71 & & \\
\hline \multirow{2}{*}{ Mejorar salud } & Riesgo & 143 & 1.59 & 0.63 & \multirow{2}{*}{1.90} & \multirow{2}{*}{ - } \\
\hline & No riesgo & 824 & 1.49 & 0.61 & & \\
\hline \multirow{2}{*}{ Estar en buena forma } & Riesgo & 144 & 1.79 & 0.50 & \multirow{2}{*}{$-5.12^{*}$} & \multirow{2}{*}{0.46} \\
\hline & No riesgo & 817 & 1.51 & 0.62 & & \\
\hline \multirow{2}{*}{ Perder peso } & Riesgo & 146 & 1.79 & 0.50 & \multirow{2}{*}{$11.77^{*}$} & \multirow{2}{*}{1.05} \\
\hline & No riesgo & 822 & 0.97 & 0.82 & & \\
\hline \multirow{2}{*}{ Quemar calorías } & Riesgo & 144 & 1.79 & 0.79 & \multirow{2}{*}{$11.82 *$} & \multirow{2}{*}{1.01} \\
\hline & No riesgo & 817 & 0.98 & 0.80 & & \\
\hline \multirow{2}{*}{ Ver amigos } & Riesgo & 140 & 1.05 & 0.78 & \multirow{2}{*}{$-2.76^{*}$} & \multirow{2}{*}{-0.26} \\
\hline & No riesgo & 819 & 1.25 & 0.76 & & \\
\hline
\end{tabular}

Nota: Significación: ${ }^{*} p<.05$

$(t=2.89 ; p<0.01 ; d=1.05)$ y quemar calorías $(t=3.05 ; p<0.01 ; d=1.01)$ que el grupo con menor riesgo. No se encontraron diferencias significativas entre ambos grupos respecto a los otros motivos (hacer ejercicio físico para divertirse, mejorar su salud, estar en buena forma o ver a los amigos).

\section{Discusión}

El propósito de este trabajo fue analizar la relación entre actividad física y riesgo de desarrollar un TA en mujeres adolescentes, la frecuencia e intensidad en su realización, el tipo de actividad física y los motivos de práctica. A pesar de que la realización de actividad física es considerada una conducta adecuada para la promoción de la salud (Castillo, Balaguer y García-Merita, 2007), los resultados de este trabajo han puesto de manifiesto que, en las 
chicas, también puede convertirse en un factor de riesgo para el desarrollo de los TA si ésta se realiza de forma excesiva y con intensidad. Estos resultados son coherentes con los obtenidos en la literatura en los que también se señala que la práctica de actividad física abusiva y como estrategia para controlar el peso puede considerarse como un factor de riesgo importante en el desarrollo de los TA (Calogero y Pedrotty, 2004; MacLean y Barr, 2003).

En referencia al tipo de actividad física, cuando diferenciamos entre la realización de ejercicio físico o deporte, los análisis no mostraron diferencias significativas entre las chicas con mayor o menor riesgo de desarrollar un TA. En cambio, cuando se diferenció entre los tipos de deportes practicados siguiendo la clasificación de Dosil (2003), no se encontraron tampoco diferencias en función del riesgo de TA, pero si en la insatisfacción corporal. Así, aquellas chicas que realizaban deportes de gimnasio presentaban mayor insatisfacción corporal que las que practicaban deportes de estética, como gimnasia rítmica y artística, patinaje, danza, etc. Estos resultados no coinciden con los del trabajo realizado por Goodwin et al. (2016), en el que encontraron diferencias significativas entre chicas que practicaban deporte y las que no, siendo las chicas que no practicaban deporte las que presentaban una mayor insatisfacción corporal. Esto puede ser debido a que, en este estudio, se analizó la diferencia en función del tipo de deporte, siendo más alta la insatisfacción corporal entre los que practicaban deporte de gimnasio, que incluso los que practicaban deportes de estética. Este dato resulta de interés, teniendo en cuenta que la insatisfacción corporal es uno de los principales factores de riesgo de TA (Alfoukha, Hamdan y Banihani, 2019).

En cuanto a los motivos de práctica de actividad física, las adolescentes con mayor riesgo de desarrollar un TA realizaban actividad física con los motivos de perder peso y quemar calorías. Estos resultados están en consonancia con la literatura previa que señala que el riesgo de desarrollar un TA se relaciona de forma positiva con practicar actividad física con el motivo de sentirse atractivo o centrado en la apariencia (Adkins y Keel, 2005; Furham et al., 2002). La actividad física "para adelgazar" se convierte así en un factor de riesgo para el desarrollo de los TA. No obstante, los resultados de este estudio no mostraron relaciones negativas entre los motivos de práctica de actividad física para divertirse o mejorar la salud con el riesgo de desarrollar un TA. En este sentido, sería necesario realizar estudios que profundizaran en la evaluación de los motivos para practicar actividad física, en función del tipo de deporte practicado. Así, en este trabajo se pone de manifiesto la importancia que tienen los motivos con los que los adolescentes practican la actividad física y el riesgo de desarrollar un TA. Todos ellos son importantes y aseguran la eficacia a la hora de llevar a cabo programas de promoción de la práctica de actividad física en las adolescentes, ya que no es la práctica de actividad física la que se relaciona con los TA, sino los motivos que les llevan a practicarla.

Entre las limitaciones del presente trabajo, estarían el propio uso de los instrumentos utilizados, todos ellos autoinformados. En futuras investigaciones, sería necesaria la evaluación del TA con instrumentos de medida específicos para detectar sus síntomas en población deportiva. Así como, puede ser clave la incorporación a la evaluación de aquellos estudiantes que practican deporte de alta competición y están federados. Otra limitación de este estudio es que se ha utilizado un diseño transversal; en el que resolver la causa y el efecto puede ser 
difícil. Las relaciones entre la insatisfacción corporal, la práctica de actividad física y sus motivos y las puntuaciones en el EAT son complejas. Por ello, sería recomendable realizar estudios longitudinales adicionales que complementasen y aclarasen estos resultados.

En conclusión, conocer la relación entre práctica de actividad física y TA, es clave para poder generar programas de prevención adecuados. Este trabajo pone de manifiesto, que más allá del tipo de actividad física que se practica, lo que se relaciona con el riesgo de desarrollar un TA son los motivos para realizarla.

\section{Referencias}

Adkins, E. C. y Keel, P. K. (2005). Does "excessive" or "compulsive" best describe exercise as a symptom of bulimia nervosa? International Journal of Eating Disorders, 38 (1), 24-29.

Alfoukha, M. M., Hamdan, A. M. y Banihani, M. (2019). Social and Psychological Factors Related to Risk of Eating Disorders Among High School Girls. Journal of School Nursing, 25, 169-177.

Allain, M. y Lentillan, V. (2016). Fitness sports, sport motives and eating disorders. Movement and Sports Science, 33, 51-63.

Alonso, J. (2006). Trastornos de la conducta alimentaria y deporte. Trastornos de la Conducta Alimentaria, 4, 368385.

Álvarez-Malé, M. L., Bautista, I. y Serra, L. (2015). Prevalencia de los trastornos de la conducta alimentaria en adolescentes de Gran Canaria. Nutrición Hospitalaria, 31(5), 2283-2288.

American Psychiatry Association. (2013). Manual diagnóstico y estadístico de los trastornos mentales (5 ed.). Madrid: Editorial Médica Panamericana.

Balaguer, I. (2002). Estilos de vida en la adolescencia. Valencia, Spain: Promolibro.

Benavente, M., Rodríguez, F., Martin, C. e Hiruela, M. V. (2003). Risk Factors Related With Eating Disorders in a Community of Adolescents. Atención Primaria, 32, 399-444.

Calogero, R. y Pedrotty, K. (2004). The practice and process of healthy exercise: an investigation of the treatment of exercise abuse in women with eating disorders. Eating Disorders, 12, 273-291.

Cantó, E. (2001). Deporte, salud, bienestar y calidad de vida. Cuadernos de Psicología del Deporte, 1 (1), 27-38.

Castillo, I., Balaguer, I. y García-Merita, M. (2007). Efecto de la práctica de actividad física y de la participación deportiva sobre el estilo de vida saludable en la adolescencia en función del género. Revista de Psicología del Deporte, 16, 201-210.

Castro, J., Toro, J., Salamero, M. y Guimera, E. (1991). The eating attitudes test: validation of the spanish versión. Psychological Assessment, 7 (2), 175-190.

Cohen, J. (1988). Statistical power analysis for the behavioural sciences. Hillsdale: Lawrence Earbaum (cit. en Pardo y San Martín, 1994).

Dosil, J. (2003). Trastornos de alimentación en deporte. Sevilla: Wanceulen.

Durán, L. J., Jiménez, P. J., Rúiz, L. M., Jiménez, F. y Camacho, M. J. (2006). Trastornos de la alimentación y deporte. Archivos de Medicina del Deporte, 23 (112), 117-125.

French, S. A., Perry, C. L., Leon, G. R. y Fulkerson, J. A. (1994). Food preferences, eating patterns, and physical activity among adolescents: correlates of eating disorders symptoms. Journal of Adolescent Health, 15 (4), 286-294.

Furnham, A., Badmin, N. y Sneade, I. (2002). Body image dissatisfaction: Gender differences in eating attitudes, selfesteem, and reasons for exercise. The Journal of Psychology, 136 (6), 581-596.

Garner, D. M. (1998). EDI-2: Inventario de Trastornos de la Conducta Alimentaria. Madrid: TEA Ediciones. 
Garner, D. M. y Garfinkel, P. E. (1979) The Eatting Attitudes Test: an index of the symptons of anorexia nervosa. Psychological Medicine, 9, 273-179.

Goodwin, H., Haycraft, E. y Meyer, C. (2016). Disordered eating, compulsive exercise, and sport participation in a UK adolescent sample. European Eating Disorders Review, 24, 304-309.

Irala, J., Cano-Prous, A., Lahortiga-Ramos, F., Gual-García, P., Martínez-González, M. A. y Cervera-Enguix, S. (2008). Validación del cuestionario Eating Attitudes Test (EAT) como prueba cribado de trastornos de la conducta alimentaria en la población general. Medicina Clínica, 130 (13), 487-491.

Kjelsas, E. y Augestad, L. B. (2004). Gender, eating behavior, and personality characteristics in physically active students. Scandinavian Journal of Medicine and Science in Sports, 14 (4), 258-268.

McLean, J. A. y Barr, S. I. (2003). Cognitive dietary restraint is associated with eating behaviors, lifestyle practices, personality characteristics and menstrual irregularity in collage women. Appetite, 40, 185-192.

Pardo, A., y San Martín, R. (1994). Análisis de datos en Psicología II. Madrid: Pirámide.

Peñas-Lledo, E., Sancho, L., y Waller, G. (2002). Eating attitudes and the use of alcohol, tobacco, and exercise among male and female adolescents. Eating Behaviors, 3, 101-111.

Thome, J. y Espelage, L. (2004). Relations among exercise, coping, disordered eating, and psychological health among college students. Eating behaviors, 5,337-341.

Wang, M. C., Walls, C. E., Peterson, K. E., Richmondo, T. K., Spadano, J., Greanay, M. L..... Bry, S. (2013). Dietary and Physical Activity Factors Related to Eating Disorder Symptoms Among Middle School Youth. Journal of School Health, 83, 14-20.

Wold, B. (1995). Health-Behavior in schoolchildren: A WHO crossnational Survey. Resource Package Questions 1993-94. Norway: University of Bergen. 